\title{
OBSERVATIONAL CONSTRAINTS ON SUBMILLIMETER DUST OPACITY
}

\author{
Yancy L. Shirley ${ }^{1,7}$, Tracy L. Huard ${ }^{2}$, Klaus M. Pontoppidan ${ }^{3,8}$, David J. Wilner ${ }^{4}$, Amelia M. Stutz ${ }^{5}$, \\ John H. BIEGING ${ }^{1}$, AND NeAL J. Evans II ${ }^{6}$ \\ ${ }^{1}$ Steward Observatory, 933 North Cherry Avenue, Tucson, AZ 85721, USA \\ 2 Astronomy Department, University of Maryland, College Park, MD 20742, USA \\ ${ }^{3}$ Caltech, Pasadena, CA, USA \\ ${ }^{4}$ Harvard-Smithsonian Center for Astrophysics, 60 Garden Street, Cambridge, MA 02138, USA \\ ${ }^{5}$ Max-Planck-Institut für Astronomie, Königstuhl 17, D-69117 Heidelberg, Germany \\ ${ }^{6}$ Astronomy Department, University of Texas at Austin, 1 University Station C1400, Austin, TX 78712-0259, USA \\ Received 2010 July 24; accepted 2010 December 16; published 2011 February 1
}

\begin{abstract}
Infrared extinction maps and submillimeter dust continuum maps are powerful probes of the density structure in the envelope of star-forming cores. We make a direct comparison between infrared and submillimeter dust continuum observations of the low-mass Class 0 core, B335, to constrain the ratio of submillimeter to infrared opacity $\left(\kappa_{\text {smm }} / \kappa_{\text {ir }}\right)$ and the submillimeter opacity power-law index $\left(\kappa \propto \lambda^{-\beta}\right)$. Using the average value of theoretical dust opacity models at $2.2 \mu \mathrm{m}$, we constrain the dust opacity at 850 and $450 \mu \mathrm{m}$. Using new dust continuum models based upon the broken power-law density structure derived from interferometric observations of B335 and the infall model derived from molecular line observations of B335, we find that the opacity ratios are $\frac{\kappa_{850}}{\kappa_{2.2}}=(3.21-4.80)_{-0.30}^{+0.44} \times 10^{-4}$ and $\frac{\kappa_{450}}{\kappa_{2.2}}=(12.8-24.8)_{-1.3}^{+2.4} \times 10^{-4}$ with a submillimeter opacity power-law index of $\beta_{\mathrm{smm}}=(2.18-2.58)_{-0.30}^{+0.30}$. The range of quoted values is determined from the uncertainty in the physical model for B335. For an average $2.2 \mu \mathrm{m}$ opacity of $3800 \pm 700 \mathrm{~cm}^{2} \mathrm{~g}^{-1}$, we find a dust opacity at 850 and $450 \mu \mathrm{m}$ of $\kappa_{850}=(1.18-1.77)_{-0.24}^{+0.36}$ and $\kappa_{450}=(4.72-9.13)_{-0.98}^{+1.9} \mathrm{~cm}^{2} \mathrm{~g}^{-1}$ of dust. These opacities are from $(0.65-0.97) \kappa_{850}^{\mathrm{OH} 5}$ of the widely used theoretical opacities of Ossenkopf and Henning for coagulated ice grains with thin mantles at $850 \mu \mathrm{m}$.
\end{abstract}

Key words: dust, extinction - ISM: clouds - stars: formation

Online-only material: color figures

\section{INTRODUCTION}

The commissioning over a decade ago of two-dimensional bolometer cameras such as SCUBA (Submillimeter Common User Bolometer Array; Holland et al. 1999) and SHARC (Submillimeter High Angular Resolution Camera; Hunter et al. 1996) permitted the efficient mapping of dust continuum emission at submillimeter wavelengths. Submillimeter continuum observations of star-forming regions in the Milky Way have constrained the physical structure of dense, star-forming cores through all embedded phases of core and protostar formation (e.g., Shirley et al. 2002; Jørgensen et al. 2002; Williams et al. 2005; Kirk et al. 2005). On larger scales, mapping of entire molecular clouds has constrained the dense core initial mass function (IMF) and made surprising connections to the shape of the stellar IMF (e.g., Motte et al. 1998, 2007; Johnstone et al. 2001; Enoch et al. 2007). In all of these studies, the mass or density scale is set by the assumed submillimeter dust opacity $\kappa\left(\mathrm{cm}^{2} \mathrm{~g}^{-1}\right)$ since the mass of optically thin submillimeter emission is inversely proportional to the dust opacity $\left(M_{d} \propto 1 / \kappa\right.$; Hildebrand 1983). It is very important to use an accurate value of the dust opacity since the mass distribution within the protostellar core directly affects the dynamical stability of the core as well as the radiative transfer through the core. The uncertainty in submillimeter dust opacity is the largest source of uncertainty in mass calculations and radiative transfer models of protostellar cores (see Shirley et al. 2005).

Since the pioneering work of Knacke \& Thomson (1973), there have been many attempts to estimate the submillimeter

\footnotetext{
7 Adjunct Astronomer, The National Radio Astronomy Observatory.

8 Hubble Fellow.
}

dust opacity in star-forming cores. The opacity of dust grains in the general interstellar medium (ISM) can vary substantially from dust opacities in environments surrounding star formation. From optical studies of dust absorption, it is well known that the ratio of total to selective extinction, $R_{V}=A_{V} / E(B-V)$, varies from $R_{V}=3.1$ in the general ISM to larger values $\left(R_{V}=5.5\right)$ in denser star-forming regions (e.g., Mathis 1990; Whittet 2003; Draine 2003). Several important physical processes directly affect the opacities in dense regions (Henning et al. 1995). The compositions of dust grains may vary from region to region. Dust grains may coagulate, changing the shape and the normalization of the general ISM size distribution (e.g., Mathis et al. 1977, 1983; Ossenkopf 1993; Ormel et al. 2009). Most of the molecular gas in a dense core is shielded from energetic photons from the forming protostar or from the interstellar radiation field (ISRF) and confined to temperatures less than 20K. Many molecular species freeze out of the gas-phase forming layers of polar $\left(\mathrm{H}_{2} \mathrm{O}\right)$ and apolar $(\mathrm{CO})$ ices that change the dielectric properties of the grains and the size distribution of grains. Theoretical calculations of the opacities make various assumptions about grain composition, grain size distributions, grain geometry, grain porosity, and ice mantle compositions; the resulting predicted submillimeter opacities vary by up to an order of magnitude (see Table 2 of Shirley et al. 2005). Currently, the most widely used calculation of opacities is that of Ossenkopf \& Henning (1994) which takes into account coagulation and varying thicknesses of ice mantles for dust grains that have persisted at high density, $n=10^{6} \mathrm{~cm}^{-3}$, for $10^{5}$ years. Even the Ossenkopf \& Henning opacities vary by a factor of a few at submillimeter wavelengths depending on the particular assumptions used in the model and there is 
no guarantee that these opacities are appropriate for the wide variety of environments toward which they have been applied, from cold, low-mass starless cores to warm, high-mass protocluster cores.

Clearly, observational constraints on submillimeter dust opacities are needed. There have been a few attempts to constrain the dust opacity at long wavelengths. These methods use observations of the amount of dust extinction at near-infrared wavelengths directly compared to the amount of dust emission at (sub)millimeter wavelengths to constrain the opacity ratio between submillimeter and near-infrared (or visible) wavelengths. Kramer et al. (1998, 2003) studied the opacity ratio in several dense starless cores in the IC 5146 region. Similarly, Bianchi et al. (2003) studied the nearby low-mass starless core B68. Both groups have demonstrated that the technique works if sensitive observations are obtained at both submillimeter and near-IR wavelengths and care is taken to compare observations taken at two very different resolutions (pencil beam at near-infrared wavelengths versus beam convolved emission at submillimeter wavelengths with non-Gaussian telescope power patterns). This method has never been applied before to a dense core which harbors a protostar.

The near-infrared to submillimeter comparison requires high signal-to-noise observations at three wavelengths (two nearinfrared wavelengths and one submillimeter wavelength). Few submillimeter studies of protostellar regions have sensitive maps that detect extended emission at high signal-to-noise ratios over large regions of the core $\left(>2^{\prime}\right)$ because the ground-based observations are limited by the size of the chopping needed for sky subtraction. One promising object is the nearby, well-studied Class 0 protostar located within the dense isolated Bok globule, Barnard 335. Since its initial detection as a far-infrared source (Keene et al. 1983), B335 has received considerable attention as it is one of the best protostellar infall candidates (Zhou et al. 1993; Choi et al. 1995; Evans et al. 2005) identified during the deeply embedded phase of low-mass star formation. For the purposes of this study, B335 has been observed with high sensitivity with SCUBA at submillimeter wavelengths (Shirley et al. 2000) and with NICMOS at near-infrared wavelengths (Harvey et al. 2001).

In this paper, we utilize a method of comparing the nearinfrared extinction to submillimeter emission to constrain the dust opacity ratio between submillimeter wavelengths and $2.2 \mu \mathrm{m}$ (Section 2) toward B335. From dust continuum radiative transfer we derive an updated physical model for B335 using currently published dust opacities (Section 3). The dust opacity ratio is determined utilizing our constraints on $T_{d}(r)$ (Section 4.1). We then constrain the opacity at submillimeter wavelengths and the opacity power-law index, $\beta_{\text {smm }}$ (Sections 4.2 and 4.3). Throughout this paper, we refer to the dust mass opacity, $\kappa_{v}$, in units of $\mathrm{cm}^{2}$ per gram of dust.

\section{METHOD}

By directly comparing infrared extinction to submillimeter emission maps along common lines of sight, we constrain the opacity ratio $\kappa_{\mathrm{smm}} / \kappa_{\mathrm{ir}}$. The emission at submillimeter wavelengths along a line of sight, $s$, is given by the equation of radiative transfer for optically thin emission, $d I_{\nu} / d \tau_{v}=B_{v}[T(s)]$ with

$$
\frac{d \tau_{\mathrm{smm}}}{d s}=\mu m_{\mathrm{H}}\left\langle m_{d} / m_{g}\right\rangle \kappa_{\mathrm{smm}}(s) n(s),
$$

where $B_{v}$ is the Planck function and $n(s)$ is the gas number density $\left(\mathrm{cm}^{-3}\right)$. We use the dust mass opacity, $\kappa_{v}\left(\mathrm{~cm}^{2} \mathrm{~g}^{-1}\right)$, where we have assumed a mean molecular weight $\mu=2.32$ and an average gas mass to dust mass ratio of $\left\langle m_{d} / m_{g}\right\rangle=1: 100$. In the derivation of the expression to determine the opacity ratio, $\mu$ and $\left\langle m_{d} / m_{g}\right\rangle$ cancel out; but they are stated explicitly here since they are used in radiative transfer modeling of the submillimeter emission (Section 3). Integrating along the line of sight gives

$$
I_{\mathrm{smm}}=\int_{s} B_{v}[T(s)] \mu m_{\mathrm{H}}\left\langle m_{d} / m_{g}\right\rangle n(s) \kappa_{\mathrm{smm}}(s) d s
$$

(Adams 1991; Shirley et al. 2000).

At infrared wavelengths, the observed intensity is due to the total amount of extinction along the line of sight,

$$
I_{\mathrm{ir}}(s)=I_{\mathrm{ir}}(0) e^{-\tau_{\mathrm{ir}}},
$$

therefore, the total extinction in magnitudes is

$$
\begin{aligned}
A_{\mathrm{ir}} & =-2.5 \log \left(\frac{I_{\mathrm{ir}}(s)}{I_{\mathrm{ir}}(0)}\right) \\
& =2.5 \log (e) \int_{s} \mu m_{\mathrm{H}}\left\langle m_{d} / m_{g}\right\rangle n(s) \kappa_{\mathrm{ir}}(s) d s .
\end{aligned}
$$

The infrared opacity includes contributions from absorption and scattering $\left(\kappa_{\mathrm{ir}}=\kappa_{\mathrm{ir}}^{\mathrm{abs}}+\kappa_{\mathrm{ir}}^{\mathrm{sca}}\right)$. Dividing Equation (2) by Equation (4), we derive the relationship between the submillimeter intensity, the infrared extinction, and the opacity ratio

$$
I_{\mathrm{smm}}=\frac{\int_{s} B_{v}[T(s)] n(s) \kappa_{\mathrm{smm}}(s) d s}{2.5 \log (e) \int_{s} n(s) \kappa_{\mathrm{ir}}(s) d s} A_{\mathrm{ir}} .
$$

If we further assume that the infrared and submillimeter opacity does not vary along the line of sight, then we find

$$
\frac{I_{\mathrm{smm}}}{P_{n}}=\left(\frac{\kappa_{\mathrm{smm}}}{\kappa_{\mathrm{ir}}}\right) A_{\mathrm{ir}},
$$

where $P_{n}$ is related to the density-weighted average Planck function and is defined as

$$
P_{n}=\frac{\int_{s} n(s) B_{v}[T(s)] d s}{2.5 \log (e) \int_{s} n(s) d s}
$$

If an isothermal approximation is used, then the column density cancels in $P_{n}$ and we are left with $P_{n}=B_{v}\left(T_{\text {iso }}\right) / 2.5 \log (e)$. However, dust temperature gradients exist throughout protostellar envelopes (Shirley et al. 2003). In this paper, we shall use the $n(r)$ and $T_{d}(r)$ determined from the best-fitted one-dimensional dust continuum radiative transfer models to calculate $P_{n}$ along each stellar line of sight.

Theoretically, the opacity ratio is determined from the slope of a plot of the submillimeter intensity versus the near-infrared extinction. In reality, neither the submillimeter intensity nor the near-infrared extinction is directly observed. Submillimeter observations actually observe the convolution of the source specific intensity distribution with the telescope beam pattern such that the observed flux density is $S_{\mathrm{smm}}=\left\langle I_{\mathrm{smm}}\right\rangle \Omega_{\text {beam }}$. A typical submillimeter beam pattern is not well described with a single Gaussian main beam as a significant fraction of the power pattern is contained within the sidelobes. The total solid angle of the beam, $\Omega_{\text {beam }}$, is determined from the integral of the normalized telescope power pattern, including sidelobes, over solid angle. 
With observations of two or more near-infrared wavelengths, we observe color differences of background stars. The extinction along a line of sight is determined from a scaling law that relates $A_{\text {ir }}$ to the observed color excess. In this paper, we will determine the extinction at the near-infrared $K$ band at $2.2 \mu \mathrm{m}$. We define the $K$-band selective extinction, $R_{K}$ such that

$$
\begin{aligned}
A_{2.2} & =R_{K}\left[(H-K)-\left\langle(H-K)_{0}\right\rangle\right] \\
& =R_{K} E(H-K)=R_{K}\left(A_{1.65}-A_{2.2}\right),
\end{aligned}
$$

where $\left\langle(H-K)_{0}\right\rangle$ is the mean intrinsic $(H-K)$ color of the background stars and $(H-K)$ is the observed infrared colors with extinction. The determination of $R_{K}$ assumes that the near-infrared extinction law is well described by a power law ( $A_{\text {ir }} \propto \lambda^{-\beta_{\text {nir }}}$ for near-infrared wavelengths; see Draine 2003, and references therein, also Flaherty et al. 2007). Chapman \& Mundy (2009) determine $\beta_{\text {nir }}=1.7$ from the slope of a plot of $E(J-H)$ versus $E(H-K)$ of background stars toward four dense cores. This is in the middle of the range of the typical values of $\beta_{\text {nir }}=1.6-1.8$ found in the literature (Draine 1989; Rieke \& Lebofsky 1985; Martin \& Whittet 1990; Whittet et al. 1993). Using this range of $\beta_{\text {nir }}$ in Equation (8), we find $R_{K}=1.59 \pm 0.12$. We shall use this value of $R_{K}$ throughout this paper.

We constrain $\kappa_{\mathrm{smm}} / \kappa_{2.2}$ from the slope of a plot of $S_{\text {smm }} / P_{n} \Omega_{\text {beam }}$ versus $E(H-K)$. If the slope is denoted by $b$, then the opacity ratio is simply $\kappa_{\mathrm{smm}} / \kappa_{2.2}=b / R_{K}$. It is important to reiterate that this derivation assumes that the dust opacity ratio along an individual line of sight is constant. If there is a variation in the dust opacity, then a linear regression is measuring an emission-weighted average dust opacity ratio along each line of sight. A monotonic change in the opacity ratio with radius will produce curvature in a plot of $S_{\text {smm }} / P_{n} \Omega_{\text {beam }}$ versus $E(H-K)$ while a distribution of opacity ratios along each line of sight will produce intrinsic scatter in the correlation.

\subsection{Submillimeter Images}

The reduction and analysis of SCUBA 850 and $450 \mu \mathrm{m}$ jiggle maps is described in detail by Shirley et al. (2000). We have re-analyzed and combined SCUBA images of B335 taken by Shirley et al. (2000) and images from the SCUBA CADC archive. B335 was observed with SCUBA in jiggle mapping mode on only two nights (1997 April 17 and 1997 December $18)$ with low atmospheric opacity $\left(\tau_{225}<0.05\right)$. Throughout this paper, for simplicity we quote the SCUBA wavelengths as 850 and $450 \mu \mathrm{m}$, whereas the actual narrowband SCUBA filters have average wavelengths of 860 and $445 \mu \mathrm{m}$, respectively. The average wavelengths are not sensitive to the shape of the source spectrum (i.e., the average wavelength changes from 860 to $859 \mu \mathrm{m}$ for sources with $v^{0}$ to $v^{4}$ ).

Before combining images from two different nights, observations of Uranus taken within $1 \mathrm{hr}$ before and after the B335 observations on each night were analyzed to compare the shape of the telescope beam pattern. We found no significant difference in the Uranus radial profiles between the two sessions. The flux density scale is calibrated using the peak and integrated Uranus flux observed on the same night as the core as determined from the FLUXES program. ${ }^{9}$ We found very good agreement in the peak flux on both nights. The specific intensities in the final image ( $\mathrm{erg} \mathrm{s}^{-1} \mathrm{~cm}^{-2} \mathrm{~Hz}^{-1} \mathrm{sr}^{-1}$ ) were estimated from the measured flux densities by using the average of the

\footnotetext{
9 http://www.jach.hawaii.edu/jac-bin/planetflux.pl
}

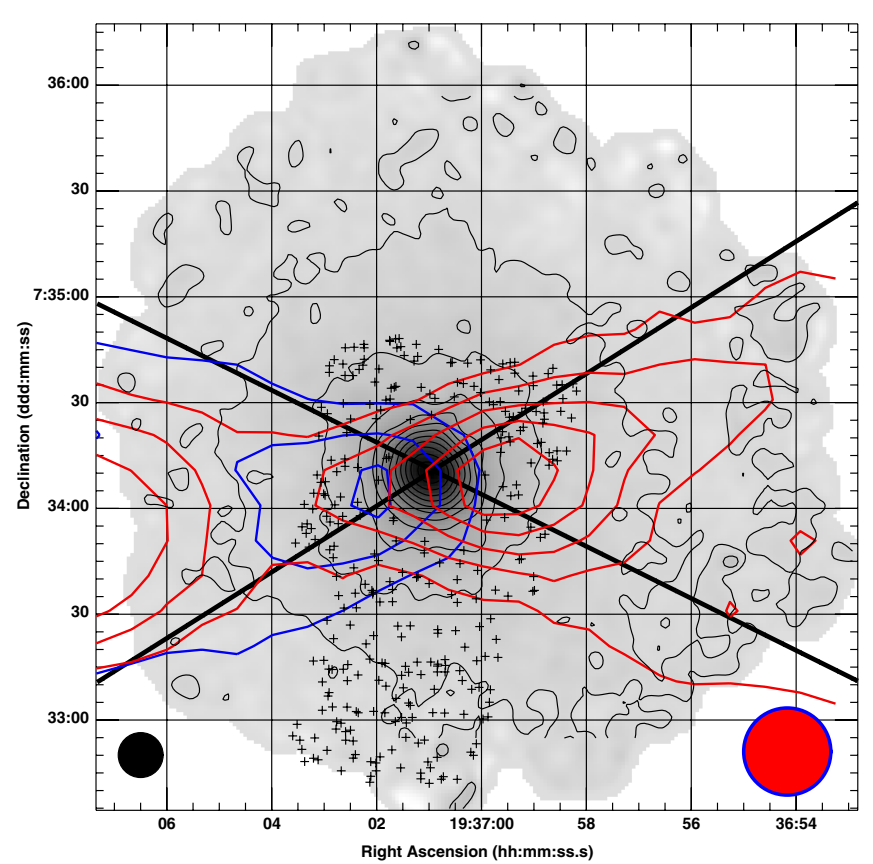

Figure 1. Gray-scale $850 \mu \mathrm{m}$ image of B335 with positions of background stars observed with NICMOS indicated by the small black crosses. All positions are in the epoch $\mathrm{J} 2000.0$. The $850 \mu \mathrm{m}$ (black) contours starts at $2 \sigma\left(30 \mathrm{mJy}\right.$ beam $\left.^{-1}\right)$ and then are spaced at $10 \%$ of the peak $\left(101 \mathrm{mJy} \mathrm{beam}^{-1}\right)$. The red and blue contours trace the outflow wings derived from CO $J=2 \rightarrow 1$ observations and start at $1.5 \mathrm{~K} \mathrm{~km} \mathrm{~s}^{-1}$ and increase by $1.5 \mathrm{~K} \mathrm{~km} \mathrm{~s}^{-1}$ (Stutz et al. 2008). The extent of the outflow cavity used to exclude background stars is shown by the two solid black lines. The SCUBA beam (lower left) and SMT CO beam (lower right) are displayed.

solid angle of telescope beam pattern determined on the same nights as the B335 observations $\left(\left\langle\Omega_{850}\right\rangle=7.3 \times 10^{-9}\right.$ sr and $\left.\left\langle\Omega_{450}\right\rangle=4.4 \times 10^{-9} \mathrm{sr}\right)$. The rms noise in the combined images is $15 \mathrm{mJy} \mathrm{beam}^{-1}$ at $850 \mu \mathrm{m}$ and $82 \mathrm{mJy} \mathrm{beam}^{-1}$ at $450 \mu \mathrm{m}$. The combined images are very similar to the published contour maps in Shirley et al. (2000; see Figure 1).

The final image pixels are oversampled $\left(1^{\prime \prime}\right)$ to determine the closest lines of sight for comparison with background stars (Figure 1). Since multiple background stars may lie within a single SCUBA beam $\left(15^{\prime \prime}\right.$ at $850 \mu \mathrm{m}$ and $8^{\prime \prime}$ at $\left.450 \mu \mathrm{m}\right)$, the submillimeter intensity will be semi-correlated in a plot of $S_{\text {smm }} / P_{n} \Omega$ versus $E(H-K)$. By using the oversampled SCUBA map, we preserve all of the information in the near-infrared extinction map. Smoothing the near-infrared line of sight to a $20^{\prime \prime}-30^{\prime \prime}$ resolution, as is typically done with extinction mapping methods (e.g., Teixeira et al. 2005; Alves et al. 2001), results in a suppression of the true scatter in line of sight $E(H-K)$. For this reason, we do not smooth the near-infrared data. Similarly, attempting to deconvolve the SCUBA beam from the B335 image is extremely difficult because the two-dimensional beam shape varies during the observations due to changes in the shape of the telescope surface and variation of parallactic angle.

\subsection{Near-infrared Images}

The near-infrared images are from NICMOS observations and are analyzed in detail by Harvey et al. (2001). Observations were performed with the NIC3 camera on the Hubble Space Telescope using the $F 160 W$ and $F 222 M$ filters. A detailed photometric comparison was made between the NICMOS magnitudes and $J$, $H$, and $K$ observations made with the NIRC camera on Keck I. B335 was imaged with a $3 \times 3$ mosaic (NIC3 field of view 
51".2) plus a $4^{\prime}$ radial strip centered on the Class 0 protostar. Background fields, off the Barnard 335 cloud, at similar galactic latitude were observed with NICMOS and NIRC. The mean intrinsic color of background stars is $\left\langle(H-K)_{0}\right\rangle=0.13 \mathrm{mag}$ and intrinsic scatter in the color is $\sigma(H-K)_{0}=0.16 \mathrm{mag}$.

The distribution of background stars compared to $850 \mu \mathrm{m}$ emission is shown in Figure 1. More than 200 background stars are observed. Most of the background stars are located outside of $20^{\prime \prime}$ with only four stars observed within $20^{\prime \prime}$ of the protostar and none within $15^{\prime \prime}$. The inability to see background stars in the innermost regions of Barnard 335 is due to the high column densities observed toward the core. Therefore, a submillimeter to near-infrared opacity comparison can only be made in the outer regions of the envelope greater than $15^{\prime \prime}$ from the protostar (3750 AU projected at a distance of $250 \mathrm{pc}$ ).

\section{AN UPDATED 1D DUST MODEL FOR B335}

The density and dust temperature along each line of sight are determined from radiative transfer models of the dust continuum emission (e.g., Shirley et al. 2003). The radiative transfer models self-consistently calculate the dust temperature profile, $T_{d}(r)$, using a one-dimensional radiative transfer code (CSDUST3; Egan et al. 1988) determined from an input density distribution $(n(r))$, ISRF, internal luminosity, and a dust opacity curve $(\kappa(\lambda))$. The model intensity profiles and spectral energy distribution are reconstructed using the same techniques as the observations (e.g., beam convolution, chopping, aperture matching). The best-fitted models minimize the $\chi_{r}^{2}$ for the observed submillimeter intensity profiles and the observed spectral energy distribution at wavelengths where the optical depth is less than unity $(\lambda>60 \mu \mathrm{m})$. Details of the radiative transfer modeling procedures for low-mass cores may be found in Shirley et al. (2002, 2005).

Unfortunately, in order to calculate $P_{n}$ and ultimately the observed opacity ratio, we have to assume a dust opacity curve to input into the radiative transfer model. Therefore, we must explore different theoretical opacities to determine how our derived opacity ratio is biased by our radiative transfer opacity choice. Since the background stars toward B335 are at projected lines of sight greater than $1000 \mathrm{AU}$ from the protostar and since B335 is a low luminosity protostar $\left(L_{\text {bol }}=3.3 L_{\odot}\right)$ embedded in a dense core that is exposed to a weaker than average ISRF (Shirley et al. 2002; Evans et al. 2005), the calculated $T(r)$ at those radii will be a slowly varying quantity with radius (Shirley et al. 2002). In this paper, we explore the effect on $T_{d}(r)$ for different dust opacity models calculated by Ossenkopf $\&$ Henning for coagulated grains with varying thicknesses of ice mantles (Columns 2, 5, and 8 of Ossenkopf \& Henning 1994) and dust opacity models calculated by Weingartner \& Draine (2001) for ISM grain populations with $R_{V}=3.1,4.0$, and 5.5 (size distribution "A" ${ }^{10}$ ). Since the original Ossenkopf \& Henning models did not calculate the scattering opacity, the $\mathrm{OH}$ opacity was divided between scattering and absorption using the ratios from the Pollack et al. (1994) models that best match the Ossenkopf \& Henning absorption opacity (Young $\&$ Evans 2005). The ratio of scattering to absorption opacities across the $3 \mu \mathrm{m}$ ice feature was determined from the albedos in Pendleton et al. (1990, Figure 4(b)) since the Pollack et al. models did not include ices. A complete explanation of the modifications to the short wavelength $\mathrm{OH}$ opacities may be

\footnotetext{
10 See http://www.astro.princeton.edu/ draine/dust/dustmix.html for the latest versions of these opacities.
}

found in Section 2.1 of Young \& Evans (2005). These short wavelength-modified $\mathrm{OH}$ opacities have been used in several published dust continuum radiative transfer calculations (e.g., Young \& Evans 2005; Shirley et al. 2005; Dunham et al. 2006, 2010).

We assume a distance of 250 pc (Tomita et al. 1979) to be consistent with previously published models, although this distance is very uncertain (see Olofsson \& Olofsson 2009). Shirley et al. (2002) explored the effects of a closer distance $(125 \mathrm{pc})$ on the radiative transfer models; however, the determination of the opacity ratio does not depend on distance.

There is also uncertainty in the modeled density structure of the B335 core. The original published dust continuum models of B335 found that a single power-law density model $(n(r) \propto$ $\left.r^{-1.8}\right)$ using $\mathrm{OH} 5$ dust opacities is a good fit to the observed intensity profile while the fit to the shape of the far-infrared SED was not as well matched (Shirley et al. 2002). Subsequently, Harvey et al. (2003a, 2003b) published an updated model based on interferometric millimeter continuum emission and nearinfrared extinction maps (Harvey et al. 2001) and found that a broken power law was a better fit to the density structure with a flatter density profile $\left(r^{-1.5}\right)$ inside $6500 \mathrm{AU}$ and steeper profile outside $\left(r^{-2.0}\right)$. Most recently, Doty et al. (2010) used broken power laws and variable opacities in four radial zones to attempt to model the large-scale dust continuum emission (excluding interferometric constraints). All of these modeling efforts found strong disagreement with the previously published molecular line radiative transfer modeling which is best fit by a Shu (1977) infall solution with a modest infall radius $r_{\text {inf }}=6200 \mathrm{AU}$ (Choi et al. 1995). Evans et al. (2005) confirmed this disagreement with updated radiative transfer modeling using non-uniform abundance profiles for several molecular species. Reconciling the modeling differences requires simultaneous modeling of molecular line and dust emission (interferometric and singledish) with varying dust opacities with radius (e.g., including $\mathrm{CO}$ desorption for $T_{d}>20 \mathrm{~K}$ ). Unfortunately, this is beyond the scope of this paper; therefore, we shall analyze the uncertainty on the opacity ratio due to our uncertainty in the underlying density model by using both the best-fit molecular line model and the best-fit dust continuum model.

We explore a grid of models that varies the dust opacity and the density scale factor, $f$. The entire density profile is scaled by a single number, $f$, to match the observed flux in a $120^{\prime \prime}$ aperture at $850 \mu \mathrm{m}$. The strength of the ISRF is constrained from molecular line modeling of $\mathrm{CO}$ observations (Evans et al. 2005). We adopt an ISRF parameterized in Shirley et al. (2005) and corresponding to $G_{0}=0.1$ Habings $\left(s_{\text {isrf }}=0.3\right.$, $\left.A_{V}\left(R_{o}\right)=1.0 \mathrm{mag}\right)$ at an outer radius of $R_{o}=3 \times 10^{4} \mathrm{AU}$, consistent with the extent of the near-infrared extinction profile (Harvey et al. 2001). Two physical models are used: a scaled Shu (1977) infall solution $\left(n(r)=f n_{\text {Shu }}(r)\right)$ with $r_{\text {inf }}=6200$ AU; and a broken power-law solution derived by Harvey et al. (2003a, 2003b),

$$
n(r)=3.3 \times 10^{4} \mathrm{fcm}^{-3}\left(\frac{r}{6500 \mathrm{AU}}\right)^{-1.5} r \in[100,6500] \mathrm{AU}
$$

$n(r)=3.3 \times 10^{4} \mathrm{fcm}^{-3}\left(\frac{r}{6500 \mathrm{AU}}\right)^{-2.0} r \in[6500,30000] \mathrm{AU}$.

Results for a subset of the models are summarized in Table 1. 
Table 1

Properties of Selected Radiative Transfer Models

\begin{tabular}{|c|c|c|c|c|c|c|c|c|c|}
\hline Physical Model $^{\mathrm{a}}$ & $\chi_{I_{450}}^{2}$ & $\chi_{I_{850}}^{2}$ & $\chi_{\mathrm{SED}}^{2}$ & $L_{>60}^{\bmod }\left(L_{\odot}\right)^{\mathrm{b}}$ & $\left\langle T_{\operatorname{los}}\right\rangle(\mathrm{K})$ & $10^{4} b_{850}{ }^{\mathrm{c}}$ & $10^{4} a_{850}{ }^{\mathrm{c}}$ & $10^{4} b_{450}{ }^{\mathrm{c}}$ & $10^{4} a_{450}{ }^{\mathrm{c}}$ \\
\hline $2.4 \times$ Harvey BPL OH $8^{d}$ & 0.36 & 0.68 & 29.55 & 3.0 & $8.88 \pm 0.73$ & $6.93 \pm 0.37$ & $0.74 \pm 0.65$ & $33.7 \pm 2.1$ & $2.3 \pm 3.7$ \\
\hline $2.8 \times$ Harvey BPL OH5 & 0.23 & 1.19 & 32.80 & 2.6 & $8.56 \pm 0.69$ & $7.63 \pm 0.40$ & $0.67 \pm 0.69$ & $39.4 \pm 2.3$ & $1.6 \pm 4.2$ \\
\hline $1.4 \times$ Harvey BPL OH2 & 0.26 & 2.01 & 43.20 & 1.8 & $8.52 \pm 0.73$ & $7.34 \pm 0.39$ & $1.18 \pm 0.68$ & $36.5 \pm 2.3$ & $6.4 \pm 4.3$ \\
\hline $2.8 \times$ Evans $\mathrm{SHU} \mathrm{OH} 8^{\mathrm{e}}$ & 4.06 & 12.37 & 32.29 & 2.7 & $9.24 \pm 0.82$ & $6.00 \pm 0.32$ & $1.11 \pm 0.56$ & $26.2 \pm 1.7$ & $5.8 \pm 3.2$ \\
\hline $11.0 \times$ Harvey BPL WD5.5 & 0.76 & 1.54 & 17.91 & 2.7 & $9.18 \pm 0.71$ & $7.61 \pm 0.37$ & $-0.75 \pm 0.65$ & $39.2 \pm 2.1$ & $-1.0 \pm 0.4$ \\
\hline $3.1 \times$ Evans SHU OH5 & 1.98 & 10.42 & 44.57 & 2.1 & $8.85 \pm 0.76$ & $6.73 \pm 0.36$ & $1.06 \pm 0.64$ & $32.0 \pm 2.0$ & $5.1 \pm 3.8$ \\
\hline $1.6 \times$ Evans $\mathrm{SHU} \mathrm{OH} 2$ & 0.75 & 6.00 & 43.41 & 1.5 & $8.91 \pm 0.84$ & $6.18 \pm 0.33$ & $1.67 \pm 0.60$ & $27.1 \pm 1.8$ & $10.8 \pm 3.5$ \\
\hline $14.0 \times$ Evans SHU WD5.5 & 0.23 & 17.91 & 37.45 & 2.3 & $9.35 \pm 0.72$ & $6.85 \pm 0.36$ & $-0.16 \pm 0.62$ & $33.4 \pm 1.9$ & $-5.2 \pm 3.3$ \\
\hline $1.0 \times$ Evans SHU OH5 & 7.72 & 15.12 & 53.49 & 1.9 & $9.74 \pm 0.93$ & $5.11 \pm 0.27$ & $1.33 \pm 0.47$ & $20.4 \pm 1.4$ & $7.0 \pm 2.7$ \\
\hline
\end{tabular}

\section{Notes.}

${ }^{a}$ Harvey BPL: Harvey et al. (2003b) broken power law $n(r)$ and Evans SHU: Evans et al. (2005) Shu-infall $n(r)$. The numbers refer to scaling factors multiplied into the density or the opacity. All models except $1.0 \times$ Evans SHU OH5 are scaled to match the observed flux at $850 \mu \mathrm{m}$.

${ }^{\mathrm{b}}$ Luminosity integrated from $\lambda \geqslant 60 \mu \mathrm{m}$. The B335 SED is published in Shirley et al. (2002) plus additional points from Spitzer Space Telescope observations at $70 \mu \mathrm{m}\left(S=15.4 \pm 2.1 \mathrm{Jy}\right.$ in a $70^{\prime \prime}$ aperture $)$ and $160 \mu \mathrm{m}\left(S=68.7 \pm 15.6 \mathrm{Jy}\right.$ in a $100^{\prime \prime}$ aperture $)$ from Stutz et al. (2008).

${ }^{c} a$ and $b$ refer to the intercept and slope of the linear regression at 850 and $450 \mu \mathrm{m}$, respectively.

d Best-fitted dust continuum model.

e Best-fitted molecular model that matches flux at $850 \mu \mathrm{m}$.

As noted by Shirley et al. (2002), the scaled Shu infall models do not fit the observed submillimeter intensity profiles or the SED. In contrast, the broken power law of Harvey et al. scaled in density by a factor of $f=2.4$ with $\mathrm{OH} 8$ opacities provides a good fit to the submillimeter intensity profiles and bolometric luminosity and a slightly better fit to the shape of the SED than the originally published best-fitted model by Shirley et al. (2002; Figure 2) which use OH5 opacities. All of the radiative transfer models have difficulty fitting the far-infrared SED indicating that there may be a problem with the theoretical opacities at those wavelengths or effects of non-spherical geometry for B335. Since the Weingartner \& Draine opacities are much smaller at submillimeter wavelengths than the Ossenkopf \& Henning opacities, the density scaling factor is higher for WD models. We calculate $P_{n}(\theta)$ for all of the models listed in Table 1 to analyze the effect of the model opacities on the derived opacity ratio (Section 4.2).

\section{RESULTS}

\subsection{Linear Regression Technique}

In order to determine the opacity ratio between a submillimeter wavelength and $2.2 \mu \mathrm{m}$, we must determine the slope from the plot of submillimeter intensity versus the near-infrared color excess. We use the Bayesian linear regression routine LINMIX_ERR (Kelly 2007) to determine the slope of a relationship of the form

$$
y=a+b x+\sigma_{\text {int }}^{2}
$$

with intrinsic scatter about the line, $\sigma_{\text {int }}^{2}$, and heteroscedastic errors in both $x$ and $y$. In our analysis, $x=E(H-K)$ and $y=$ $S_{\text {smm }} / P_{n} \Omega_{\text {beam }}$. LINMIX_ERR approximates the distribution of the independent variable $(x)$ as a mixture of Gaussians. This method alleviates the ad hoc assumption of a uniform prior distribution on the independent variable that is used in the derivation of popular $\chi^{2}$ minimization routines such as XYEFIT (e.g., Press et al. 1992; Tremaine et al. 2002; Weiner et al. 2006) and also permits fitting of truncated data sets (e.g., Malmquist bias) and data sets that include censored data or upper limits. Details of the assumed prior distributions are described in detail in Kelly (2007). Direct computation of the posterior distribution is too computationally intensive; therefore, random draws from the posterior distribution are obtained using a Markov Chain Monte Carlo method (Metropolis-Hastings Algorithm; Chib \& Greenberg 1995; Tierney 1998; Gelman et al. 2004). We fit our data using the publicly available IDL code LINMIX_ERR.pro (Kelly 2007) to determine the distributions of $a, b$, and $\sigma_{\text {int }}^{2}$. In order to test the robustness of the resulting distributions, we varied the number of Gaussians from $k=2$ to $k=4$ and used various numbers of iterations up to $10^{4}$.

The plots of $S_{\mathrm{smm}} / P_{n} \Omega_{\text {beam }}$ versus $E(H-K)$ are shown in Figures 3 and 4 for the best-fitted dust continuum model $(2.4 \times$ Harvey BPL OH8) at $850 \mu \mathrm{m}$ and $450 \mu \mathrm{m}$. The histograms of the slope and intercept are well approximated by Gaussian distributions (see the inset in Figures 3 and 4); therefore, we tabulate the mean and standard deviations of the slope and intercept distributions (Table 1). The linear regression is only performed on data points that lie outside the outflow cavities. Harvey et al. (2001) noticed a bimodal distribution of color excesses depending on whether a background star was located in the direction of the east-west-oriented molecular outflow cavity. The opening angle of the $\mathrm{CO}$ outflow was recently characterized by Stutz et al. (2008) to be $55^{\circ}$. This is slightly larger than the outflow opening angle of $40 \pm 5^{\circ}$ used in Harvey et al. (2001, 2003a). Since we are making a comparison of the opacity properties of the dust in the envelope, and since shock processing of dust within the outflow cavity walls may affect the grain opacities, we use the larger estimate for the outflow opening angle $\left(55^{\circ}\right)$ in this paper.

\subsection{Opacity Ratios and Submillimeter Opacity}

The opacity ratio is determined from plots similar to Figures 3 and 4 for all of the models discussed in Section 3. Linear regression from the LINMIX_ERR method provides good fits to the observed correlations. We find no evidence for large, systematic curvature in the plots of $S_{\text {smm }} / P_{n} \Omega$ versus $E(H-K)$ indicating that over the range of impact parameters probed by background stars $\left(\theta \in\left[15^{\prime \prime}, 70^{\prime \prime}\right]\right)$, there is not a strong monotonic gradient in the intensity-weighted dust opacity with radius. This result independently confirms the conclusions by Shirley et al. (2002) that there is no evidence for large-scale opacity changes from comparisons of 850 and $450 \mu \mathrm{m}$ model 

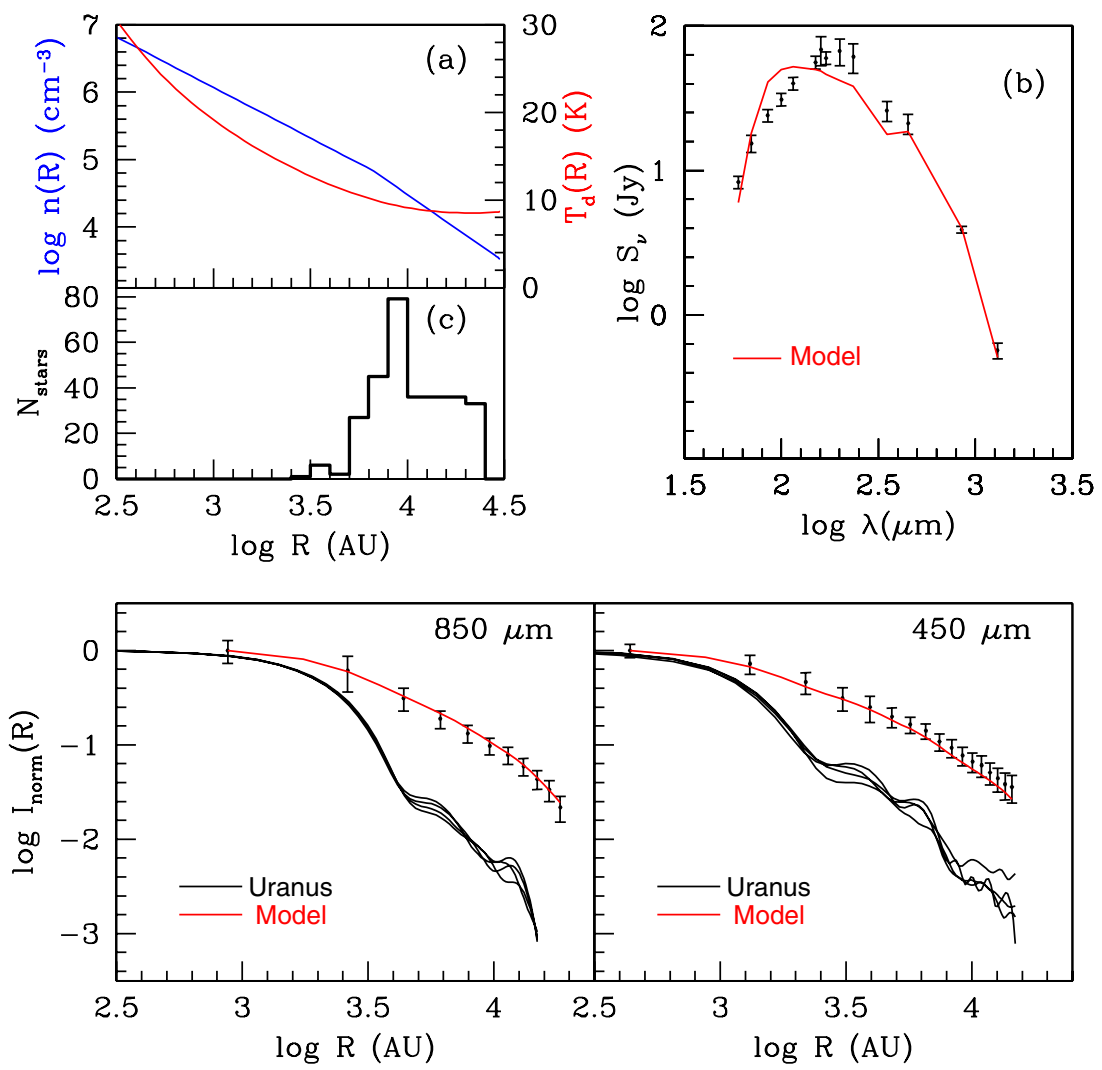

Figure 2. Updated best-fit dust model for B335, 2.4×Harvey BPL OH8. Panel (a) displays the scaled-Harvey broken power-law density profile (blue) and the resulting dust temperature profile (red). The fit to the SED is shown in panel (b). The histogram showing the location of background stars that are used to constrain the opacity ratio is shown in panel (c). The distribution is strongly peaked just below $10^{4} \mathrm{AU}$. The fit to the submillimeter intensity profiles at 850 and $450 \mu \mathrm{m}$ is shown in the bottom panels. The red curves are the dust model profiles while the black curves are the beam profiles determined from Uranus observations bracketing the B335 observations. The intensity error bars account for statistical uncertainty in the intensity as well as azimuthal variations in intensity within each annulus.

(A color version of this figure is available in the online journal.)

intensity profiles. This result is also consistent with the findings of Doty et al. (2010) which indicate nearly constant dust opacities over the range of radii that are fit in this paper. A caveat is that there is significant scatter in the correlations at both submillimeter wavelengths that could mask opacity changes. The scatter appears to become slightly larger near the highest $E(H-K)$ observed. This may indicate that opacities are beginning to change within a few thousand $\mathrm{AU}$ of the protostar. Theoretically, we expect changes in the ice mantle composition as desorption due to protostellar heating occurs in the inner envelope. How this affects the observed dust opacity is still unknown. Unfortunately, NICMOS was not sensitive enough to detect to detect background stars within $10^{\prime \prime}$ of the protostar.

The underlying density and temperature distribution affect the slope of the correlation through $P_{n}$. In order to analyze the effect of the temperature profile on the opacity ratio, we first assume an isothermal approximation where every line of sight is assumed to have the same dust temperature $(T(r)=$ constant). The calculated opacity ratios for isothermal temperatures are shown as the solid curve in Figure 5. For example, the $850 \mu \mathrm{m}$ ratio varies by a factor of 2.5 for dust temperatures from 7 to $14 \mathrm{~K}$. In reality, each line of sight through the protostellar envelope is non-isothermal (Section 3). We calculate $P_{n}(\theta)$ from Equation (7) using the density $(n(r))$ and dust temperature profiles $\left(T_{d}(r)\right)$ determined from the dust radiative transfer models for each line of sight $(\theta)$. For each of the dust continuum models, we estimated the isothermal temperature through the stellar lines of sight used in the linear regression by solving
Equation (7) for the temperature and using the calculated lineof-sight $P_{n}(\theta)$ from the radiative transfer model

$$
T_{\mathrm{los}}(\theta)=\frac{h v / k}{\ln \left(1+\frac{2 h v^{3}}{2.5 \log _{10}(e) c^{2} P_{n}(\theta)}\right)} .
$$

This temperature corresponds to the single temperature that characterizes a non-isothermal line of sight. The average $\left\langle T_{\text {los }}\right\rangle$ is calculated by averaging $T_{\text {los }}$ for the 190 lines of sight used in the linear regression (Section 4.1). When the more realistic $T(r)$ from the radiative transfer models is included in the regression, then the calculated opacity ratio is always below the opacity ratio determined from isothermal lines of sight (Figure 5). This is a systematic effect caused by a monotonically decreasing temperature profile $(d T / d r<0$; see Figure 2(a)) along each line of sight. It is very important to account for temperature gradients when determining the opacity ratio in the envelopes of Class 0 protostars.

Figure 5 also graphically illustrates the range of uncertainty introduced into the determination of $\kappa_{\mathrm{smm}} / \kappa_{\text {ir }}$ due to different model opacity assumptions and different physical models. In quoting our opacity ratio, we choose two limiting models that characterize the range of $\kappa_{\mathrm{smm}} / \kappa_{\mathrm{ir}}$ and which also fit the observed $850 \mu \mathrm{m}$ flux. Those two models are $2.8 \times$ Harvey BPL OH5 and $2.8 \times$ Evans Shu OH8 in Table 1 . We find that $\frac{\kappa_{850}}{\kappa_{2.2}}=$ $(3.21-4.80)_{-0.30}^{+0.44} \times 10^{-4}$ and $\frac{\kappa_{450}}{\kappa_{2.2}}=(12.8-24.8)_{-1.3}^{+2.4} \times 10^{-4}$, where the range in $\kappa_{\text {smm }} / \kappa_{\text {ir }}$ corresponds to the value for each physical model. 


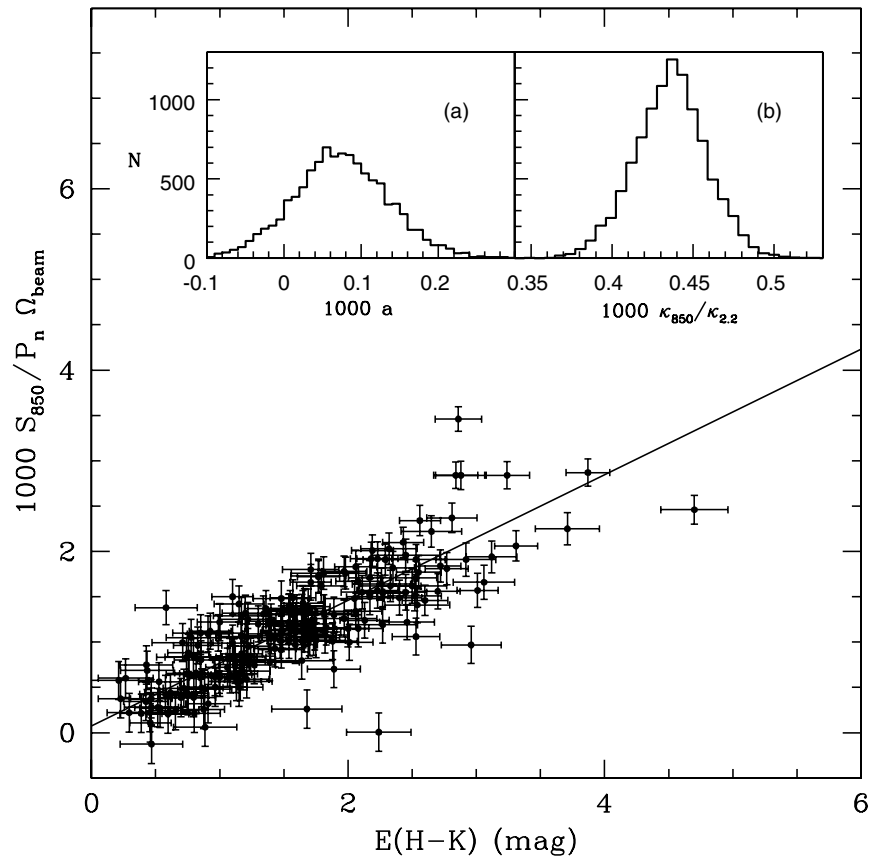

Figure 3. $850 \mu \mathrm{m}$ intensity plotted vs. $(H-K)$ color excess. $P_{n}$ is related the density-weighted Planck function. The solid line is the linear regression with the mean slope and intercept from the Posterior distributions. Histograms of the intercept (a) and slope (b) distributions are shown in the insets. $\kappa_{850} / \kappa_{2.2}=b_{850} / R_{K}$ where we have assumed $R_{K}=1.59$ (see Section 2).

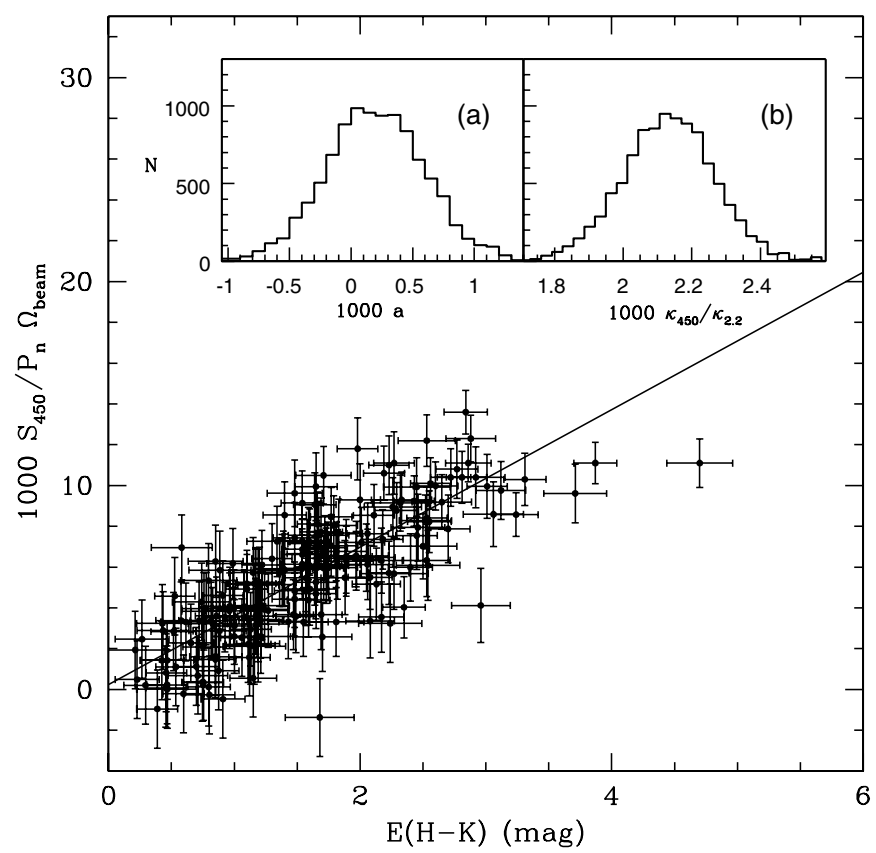

Figure 4. $450 \mu \mathrm{m}$ intensity plotted vs. $(H-K)$ color excess. $P_{n}$ is related to the density-weighted Planck function. The solid line is the linear regression with the mean slope and intercept from the Posterior distributions. Histograms of the intercept (a) and slope (b) are shown in the insets. $\kappa_{450} / \kappa_{2.2}=b_{450} / R_{K}$ where we have assumed $R_{K}=1.59$ (see Section 2).

We may compare our value of the opacity ratio with previous determinations toward dense cores (Figure 6). Bianchi et al. (2003) observed the starless core B68 at 850 and $1200 \mu \mathrm{m}$ with SCUBA and SIMBA. They employ a similar technique to compare the submillimeter intensity and near-infrared colors. However, they assume isothermality of the dust temperature and the extinction law of Rieke \& Lebofsky (1985). Since they

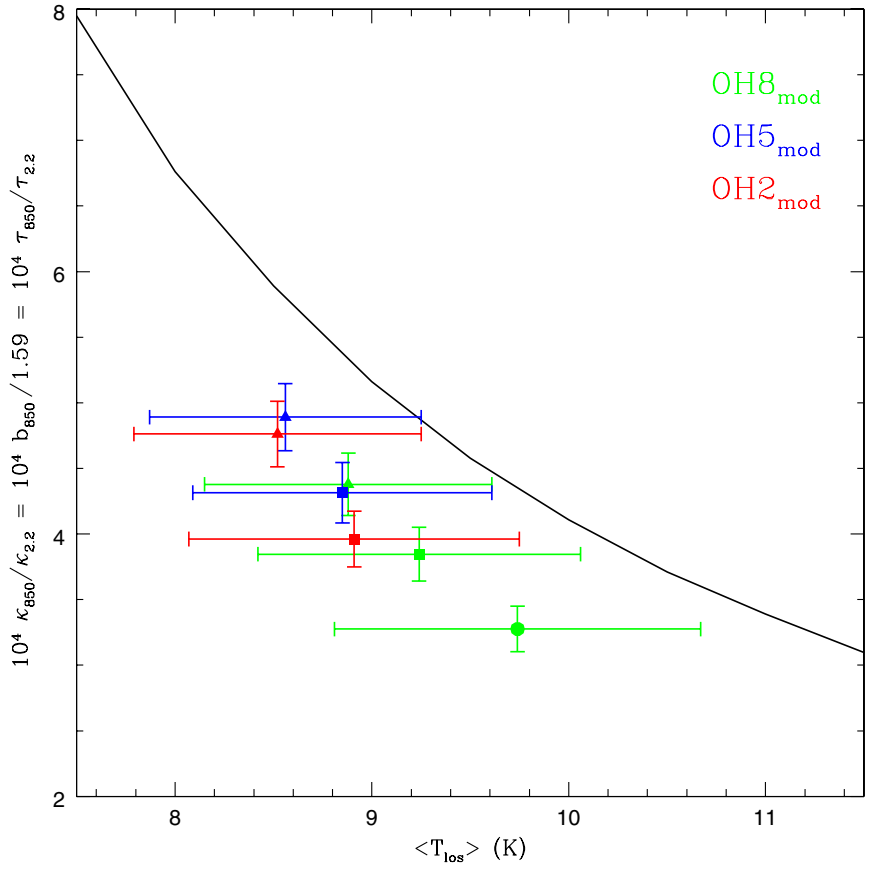

Figure 5. $\kappa_{850} / \kappa_{2.2}$ vs. the average line-of-sight dust temperature. $\kappa_{850} / \kappa_{2.2}$ is determined from the slope of linear regressions $\left(b_{850}\right.$ see Figures 3 and 4 ) divided by $R_{K}=1.59$ (see Section 2 ) for different physical models $(n(r), T(r))$. The solid line is for dust models with an isothermal envelope $(T(r)=$ constant). The plotted points are for various dust models with calculated $T(r)$. Symbols refer to scaled Harvey broken-power law (triangles), scaled Shu model (squares), and Evans-Shu model (circle). Color indicates the dust opacity model used to calculate $T(r)$ : $\mathrm{OH} 8_{\text {mod }}$ (green), $\mathrm{OH} 5_{\text {mod }}$ (blue), and $\mathrm{OH} 2_{\text {mod }}$ (red) (Section 3). All models except for the Evans-Shu model are scaled in density to match the observed $850 \mu \mathrm{m}$ flux.

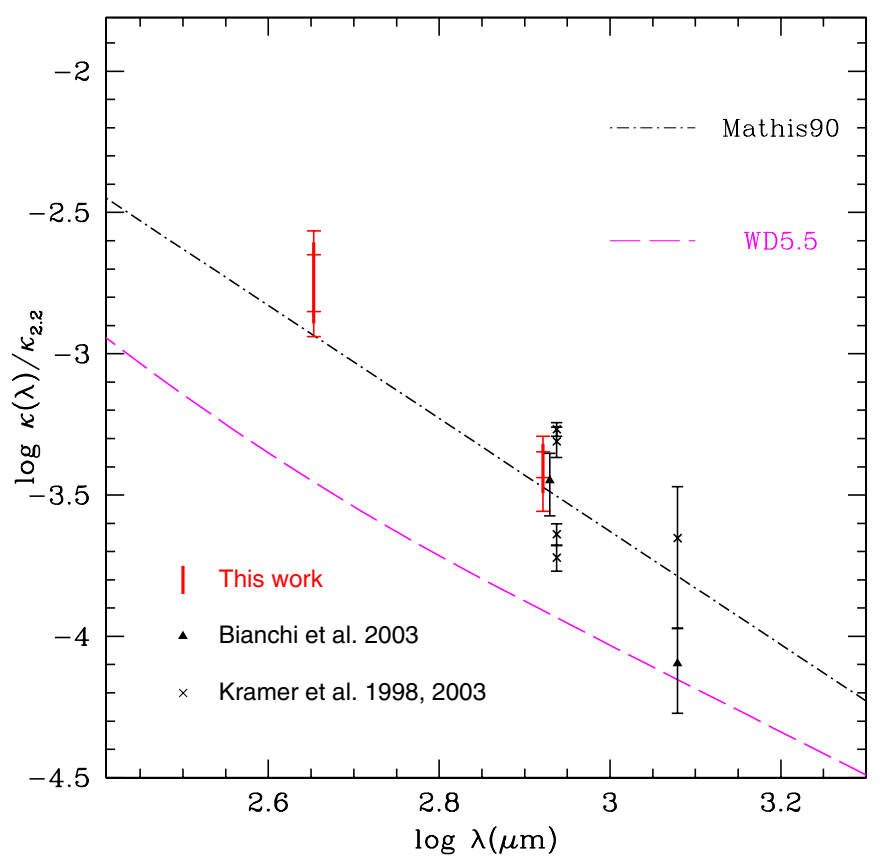

Figure 6. Theoretical kappa ratios with the observed opacity ratios from this work (B335, shown in red), Bianchi et al. (B68, 2003), and Kramer et al.

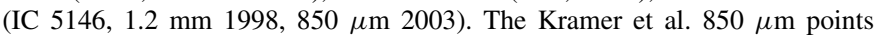
have been shifted slightly in wavelength for clarity. WD: Weingartner \& Draine (2001) for $R_{V}=5.5$. Mathis refers to the parameterization by Mathis (1990) of the ISM empirical dust model. OH model profiles are not included in this figure since self-consistent scattering opacities were not determined by Ossenkopf \& Henning (1994) 


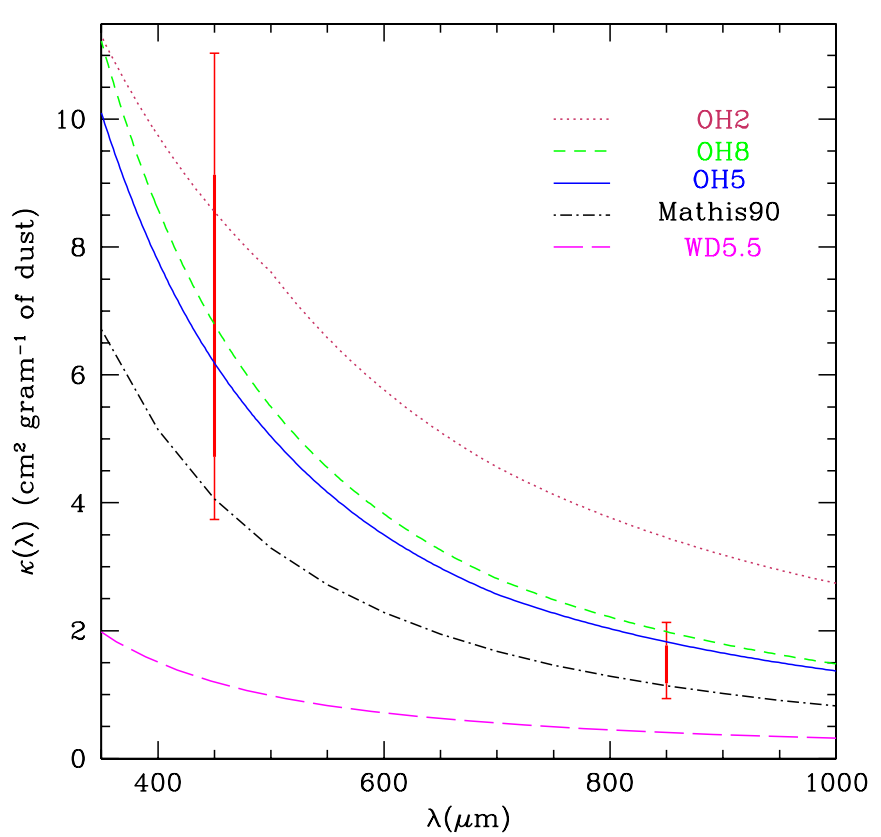

Figure 7. Our constraints on the submillimeter dust opacity assuming and absolute value of the opacity at $2.2 \mu \mathrm{m}$ of $3800 \pm 700 \mathrm{~cm}^{2} \mathrm{~g}^{-1}$. WD: Weingartner \& Draine (2001) for $R_{V}=5.5$. OH: Ossenkopf \& Henning (1994) for (2) no ice mantles, (5) thin ice mantles, and (8) thick ice mantles (note the Young \& Evans 2005 modification to the $\mathrm{OH}$ opacities does not affect the submillimeter opacities). Mathis refers to the parameterization by Mathis (1990) of the ISM empirical dust model.

(A color version of this figure is available in the online journal.)

determine the opacity ratio with respect to the opacity at optical wavelength, $V$, we must use the Rieke \& Lebofsky $A_{K} / A_{V}=$ $1 / 8.9$ to convert from $\kappa_{V}$ to $\kappa_{2.2}$. The resulting opacity ratios are $\kappa_{850} / \kappa_{2.2}=3.6 \pm 0.9 \times 10^{-4}$ and $\kappa_{1200} / \kappa_{2.2}=8.0 \pm 2.7 \times 10^{-5}$. The $850 \mu \mathrm{m}$ ratio is comparable to the ratio we determined for B335.

Similarly, Kramer et al. (2003) determined the $850 \mu \mathrm{m}$ opacity ratio toward four cores in the IC 5146 filament. This study includes an analysis of dust temperature variations between the cores in the filament. Again, we must use the Rieke \& Lebofsky $A_{K} / A_{V}$ to convert to $\kappa_{2.2}$. The four cores have $\kappa_{850} / \kappa_{2.2}$ that range from $1.9 \pm 0.2 \times 10^{-4}$ to $5.4 \pm 0.3 \times 10^{-4}$. Kramer et al. also find evidence that the opacity ratio has an inverse dependence on the dust temperature (see Figure 5). The $\left\langle\kappa_{850} / \kappa_{2.2}\right\rangle$ from all three studies is $3.7 \pm 1.4 \times 10^{-4}$. Unfortunately, no opacity ratios have been determined previously in the literature at $450 \mu \mathrm{m}$ toward low-mass dense cores.

Converting the observed opacity ratio to the submillimeter opacity requires an estimate of the opacity at $2.2 \mu \mathrm{m}$. A first approximation is to average the $\kappa_{2.2}$ from many different theoretical opacity models. Averaging the $2.2 \mu \mathrm{m}$ opacities from the Ossenkopf \& Henning models (OH2, OH5, OH8; 1994), the opacities used in the multi-dimensional dust models of B. A. Whitney et al. (2007, private communication; e.g., Whitney et al. 2003), the Mathis et al. opacity (1983), and the new theoretical opacities calculated by K. Pontoppidan (2007, private communication) that match the cores-to-disk midinfrared extinction law and ice features (K. Pontoppidan et al. 2011 , in preparation), we find $\left\langle\kappa_{2.2}\right\rangle=3800 \pm 700 \mathrm{~cm}^{2} \mathrm{~g}^{-1}$ of dust. Multiplying this number into the opacity ratios results in the submillimeter opacities of $\kappa_{850}=(1.18-1.77)_{-0.24}^{+0.36}$ and $\kappa_{450}=(4.72-9.13)_{-0.98}^{+1.9} \mathrm{~cm}^{2} \mathrm{~g}^{-1}$ of dust. These opacities are plotted with theoretical curves in Figure 7 . We note that this crude average for $\kappa_{2.2}$ results in a large error bar in the calculated 850 and $450 \mu \mathrm{m}$ opacities because the $2.2 \mu \mathrm{m}$ opacities vary by a factor of two among the different theoretical models.

The uncertainty in the 850 and $450 \mu \mathrm{m}$ opacity ratios and opacities make our determinations consistent with the empirical opacity law parameterized by Mathis $(1990 ; \kappa=$ 13.16 $(\lambda / 250 \mu \mathrm{m})^{-2}$; also parameterized in Kramer et al. 2003) as well as the coagulated dust model with thin ice mantles of Ossenkopf \& Henning (1994, OH5). The opacity model, OH8, that provides the best fit to the submillimeter and intensity profile and SED is at the upper statistical error bar at $850 \mu \mathrm{m}$ and the lower bound of the models at $450 \mu \mathrm{m}$. Our results bracket the popular theoretical opacities (OH5) at $850 \mu \mathrm{m}$ that have been used in dust continuum radiative transfer modeling (e.g., Shirley et al. 2002, 2005; Mueller et al. 2002; Young et al. 2004; Dunham et al. 2006; Doty et al. 2010).

\subsection{Power-law Index $\beta$}

At far-infrared and submillimeter wavelengths $>100 \mu \mathrm{m}$, the opacity falls as a power law with increasing wavelength $\left(\kappa(\lambda) \propto \lambda^{-\beta_{\text {smm }}}\right)$. Estimating $\beta_{\text {smm }}$ is a difficult problem. The most traditional methods have used modified blackbody fits to the SED or ratios of submillmeter wavelengths to constrain $\beta_{\text {smm }}$ (e.g., Visser et al. 1998; Shirley et al. 2000). Both of these methods assume a single dust temperature which is not an appropriate assumption for Class 0 protostars which have strong temperature gradients (i.e., Figure 2). Instead, we use the derived opacity ratios at two submillimeter wavelengths to constrain $\beta_{\text {smm }}$ between 450 and $850 \mu \mathrm{m}$. Since this method utilizes background stars that are at least $15^{\prime \prime}$ from the central protostar, the opacity ratios are probing the dust properties in the outer, cold portions of the envelope. This differs from the previous two methods which use fluxes that include significant contributions from warmer dust near the protostar. The opacity ratio $\beta_{\text {smm }}$ is given by

$$
\beta_{\mathrm{smm}}=\frac{\ln \left(\frac{R_{K} \kappa_{450} / \kappa_{2.2}}{R_{K} \kappa_{850} / \kappa_{2.2}}\right)}{\ln (850 / 450)}=1.572 \ln \left(\frac{b_{450}}{b_{850}}\right) .
$$

Since this is a ratio, the exact value of $R_{K}$ cancels; however, the ratio is still sensitive to systematic uncertainties such as flux calibration errors. The opacity ratio for B335 is $\beta_{\mathrm{smm}}=$ $(2.18-2.58)_{-0.30}^{+0.30}$.

If we compare our results to Bianchi et al. (2003) which determined the opacity ratio at 850 and $1200 \mu \mathrm{m}$, we find a severe discrepancy that illustrates the importance of the calibration in determining $\beta$. The Bianchi $\beta_{\mathrm{smm}}=4.3 \pm 1.3$ is much higher than our $\beta_{\mathrm{smm}}$. This anomalous result indicates a systematic calibration problem at one or both wavelengths. Great care must be taken when comparing observations made with different instruments on different telescopes through different observing conditions (e.g., SCUBA and SIMBA). Observations taken with SCUBA simultaneously at 850 and $450 \mu \mathrm{m}$ avoid this problem since the observations are taken in the same atmospheric conditions. While a calibration error at 450 or $850 \mu \mathrm{m}$ could account for our $\beta_{\text {smm }}>2$, we have taken great care to assure a stable calibration between 850 and $450 \mu \mathrm{m}$ by comparing the flux calibration of Uranus taken on several nights surrounding the B335 observations. The ratio of the $450-850 \mu \mathrm{m}$ flux conversion factors (see Jenness et al. 2002) never vary by more than $10 \%$ during these time periods.

Observational evidence for submillimeter opacity indices above two in the ISM exists. A detailed multi-wavelength study 
of the starless core, TMC-1C, using multiple methods to determine the opacity index finds that $1.7 \leqslant \beta \leqslant 2.7$ with a most likely value near $\beta=2.2$ (Schnee et al. 2010). Another example is the PRONAOS (PROgramme NAtional d'Observations Submillimértiques; Lamarre et al. 1994) balloon-borne experiment which finds that the opacity index has an inverse temperature dependence with $\beta>2$ for $T<12.7 \mathrm{~K}$ (Dupac et al. $2003)$. The lower bound of our opacity index overlaps with the PRONAOS opacity index curve $(\beta=1 /(0.40+0.0079 T)$; Dupac 2009) for the typical $\left\langle T_{\text {los }}\right\rangle$ found in our dust continuum models (see Figure 5). The PRONAOS results are not unique as an inverse temperature dependence of $\beta$ and opacity indices greater than two at low temperatures has also been seen in farinfrared and submillimeter observations from the ARCHEOPS balloon-borne experiment (Désert et al. 2008).

The tendency of the opacity law toward $\beta=2$ was noted from early submillimeter observations and is thought to originate from behavior of the complex dielectric function $\epsilon(\operatorname{Re}(\epsilon)$ $=$ const, $\operatorname{Im}(\epsilon) \propto \nu$; Wickramasinghe 1967) of the grains at wavelengths far from resonances in the grain materials (e.g., Gezari et al. 1973). None of the popular opacity models used in protostellar dust continuum modeling or modeling of ISM dust predict power-law indices greater than two (i.e., $\mathrm{OH} 2$ $\beta_{\mathrm{smm}}=1.35$, OH5 $\beta_{\mathrm{smm}}=1.85$, OH8 $\beta_{\text {smm }}=1.88$, WD5.5 $\beta_{\text {smm }}=1.69$; see Shirley et al. 2005). While our individual submillimeter opacity constraints overlap the OH5 model at both 450 and $850 \mu \mathrm{m}$ due to the uncertainty in the physical model that best fits B335, the opacity index must be determined using the same physical model at both wavelengths and the resulting $\beta_{\text {smm }}$ is too steep to be consistent with the $\mathrm{OH} 5$ model.

There is a class of amorphous silicate dust models which include phonon difference processes (disordered charge distributions and localized two level systems; see Schlömann 1964; Phillips 1987) that result in $\beta_{\text {submm }}>2$ at low temperatures (e.g., Meny et al. 2007). These processes have been used to explain the anti-correlation between $\beta_{\text {smm }}$ and temperature observed by the PRONAOS experiment (Boudet et al. 2005). Our $\beta_{\text {smm }}$ range is consistent with the predicted opacity index from Boudet et al. (2005) and Meny et al. (2007) for the typical $T_{\text {los }}<10 \mathrm{~K}$ derived from the dust models. Ultimately, our results should be tested by reproducing this analysis for observations with the new generation of submillimeter cameras (e.g., SPIRE, LABOCA, SCUBA2) and using better constraints from more sophisticated (e.g., multi-dimensional) dust continuum models of B335.

\section{CONCLUSIONS}

We have determined the opacity ratio from the slope of a plot of submillimeter intensity versus near-infrared color excess toward B335. The submillimeter intensity along each line of sight in the correlation is corrected for the non-isothermal temperature profile by the quantity $1 / P_{n}$ which is related to the density-weighted average Planck function. We find opacity ratios of $\frac{\kappa_{850}}{\kappa_{22}}=(3.21-4.80)_{-0.30}^{+0.44} \times 10^{-4}$ and $\frac{\kappa_{450}}{\kappa_{22}}=$ $(12.8-24.8)_{-1.3}^{+2.4} \times 10^{-4}$ for a ratio of total to selective $K$ band extinction of $R_{K}=1.59 \pm 0.12$. The range in values corresponds to the uncertainty in the physical model for the envelope of B335. The submillimeter opacity power-law index is $\beta_{\mathrm{smm}}=(2.18-2.58)_{-0.30}^{+0.30}$. For an average $2.2 \mu \mathrm{m}$ opacity of $3800 \pm 700 \mathrm{~cm}^{2} \mathrm{~g}^{-1}$, we find an opacity at 850 and $450 \mu \mathrm{m}$ of $\kappa_{850}=(1.18-1.77)_{-0.24}^{+0.36}$ and $\kappa_{450}=(4.72-9.13)_{-0.98}^{+1.9} \mathrm{~cm}^{2} \mathrm{~g}^{-1}$. These opacities statistically agree with the popular theoretical ratios of Ossenkopf and Henning for coagulated ice grains with thin mantles $(0.65-0.97) \kappa_{850}^{\mathrm{OH} 5}$ at $850 \mu \mathrm{m}$; however, our derived opacity index $\left(\beta_{\mathrm{smm}}\right)$ is steeper than predicted by the OH5 model $\left(\beta_{\mathrm{OH} 5}=1.85\right)$. This comparison of near-infrared color excess and submillimeter emission probes the opacity on scales of $15^{\prime \prime}$ to $75^{\prime \prime}$, and does not find evidence for a large-scale variation in the opacity on those scales. We confirm a disagreement between the best-fitted dust radiative transfer model and the best-fitted molecular line radiative transfer model. Improvements in the estimate of the opacity ratios and submillimeter opacities may be made with more sophisticated, multi-dimensional modeling of the dust continuum emission such as variable dust opacities in the inner envelope where desorption of $\mathrm{CO}$ and other molecules may change the optical constants of grains. The techniques used in this analysis should be applicable to farinfrared and submillimeter observations of B335 with the Herschel Space Observatory. With the commissioning of new, sensitive bolometer cameras, such as LABOCA and SCUBA-2, combined with observations with large format infrared CCDs on large aperture telescopes (JWST, Keck, etc.), it will be possible to extend this method to study the dust opacity ratio around other Class 0 protostars.

We sincerely thank the referee for a very thorough and careful reading of the manuscript. We thank Brandon Kelly for comments that improved this paper. Guest User, Canadian Astronomy Data Centre, is operated by the Dominion Astrophysical Observatory for the National Research Council of Canada's Herzberg Institute of Astrophysics. K.M.P. is supported by NASA through Hubble Fellowship grant no. 01201.01 awarded by the Space Telescope Science Institute, which is operated by the Association of Universities for Research in Astronomy, Inc., for NASA, under contract NAS 5-26555. Additional support came from NASA Origins grant NNG04GG24G and the Spitzer Legacy Science Program, provided by NASA through contracts 1224608 and 1230779 issued by the Jet Propulsion Laboratory, California Institute of Technology, under NASA contract 1407 to N.J.E.

\section{REFERENCES}

Adams, F. C. 1991, ApJ, 382, 544

Alves, J. F., Lada, C. J., \& Lada, E. A. 2001, Nature, 409, 159

Akritas, M. G., \& Bershady, M. A. 1996, ApJ, 470, 706

Bianchi, S., Gonçalves, J., Albrecht, M., Caselli, P., Chini, R., Galli, D., \& Walmsley, M. 2003, A\&A, 399, L43

Boudet, N., Mutschke, H., Nayral, C., Jäger, C., Bernard, J.-P., Henning, T., \& Meny, C. 2005, ApJ, 633, 272

Chapman, N. L., \& Mundy, L. G. 2009, ApJ, 699, 1866

Chib, S., \& Greenberg, E. 1995, Am. Stat., 49, 327

Choi, M., Evans, N. J., II, Gregersen, E. M., \& Wang, Y. 1995, ApJ, 448, 742 Désert, F.-X., et al. 2008, A\&A, 481, 411

Doty, S. D., Tidman, R., Shirley, Y., \& Jackson, A. 2010, MNRAS, 406, 1190

Draine, B. T. 1989, in Proc. 22nd Eslab Symp., Infrared Spectroscopy in Astronomy, ed. B. H. Kaldeich (ESA SP-290; Noordwijk: ESA), 93

Draine, B. T. 2003, ARA\&A, 41, 241

Dunham, M. M., Evans, N. J., II, Terebey, S., Dullemond, C. P., \& Young, C. H. 2010, ApJ, 710, 470

Dunham, M. M., et al. 2006, ApJ, 651, 945

Dupac, X. 2009, in ASP Conf. Ser. 414, Cosmic Dust-Near and Far, ed. T. Henning, Grün E., \& J. Steinacker (San Francisco, CA: ASP), 372

Dupac, X., et al. 2003, A\&A, 404, L11

Egan, M. P., Leung, C. M., \& Spagna, G. R. 1988, Comput. Phys. Commun., 48, 271

Enoch, M. L., Glenn, J., Evans, N. J., II, Sargent, A. I., Young, K. E., \& Huard, T. L. 2007, ApJ, 666, 982

Evans, N. J., II, Lee, J.-E., Rawlings, J. M. C., \& Choi, M. 2005, ApJ, 626, 919

Flaherty, K. M., Pipher, J. L., Megeath, S. T., Winston, E. M., Gutermuth, R. A., Muzerolle, J., Allen, L. E., \& Fazio, G. G. 2007, ApJ, 663, 1069 
Gelman, A., Carlin, J. B., Stern, H. S., \& Rubin, D. B. 2004, Bayesian Data Analysis (2nd ed.; Boca Raton, FL: Chapman \& Hall)

Gezari, D. Y., Joyce, R. R., \& Simon, M. 1973, ApJ, 179, L67

Harvey, D. W. A., Wilner, D. J., Lada, C. J., Myers, P. C., Alves, J. F., \& Chen, H. 2001, ApJ, 563, 903

Harvey, D. W. A., Wilner, D. J., Myers, P. C., \& Tafalla, M. 2003a, ApJ, 596, 383

Harvey, D. W. A., Wilner, D. J., Myers, P. C., Tafalla, M., \& Mardones, D. 2003b, ApJ, 583, 809

Henning, T., Michel, B., \& Stognienko, R. 1995, Planet. Space Sci., 43, 1333

Hildebrand, R. H. 1983, QJRAS, 24, 267

Holland, W. S., et al. 1999, MNRAS, 303, 659

Hunter, T. R., Benford, D. J., \& Serabyn, E. 1996, PASP, 108, 1042

Jenness, T., Stevens, J. A., Archibald, E. N., Economou, F., Jessop, N. E., \& Robson, E. I. 2002, MNRAS, 336, 14

Johnstone, D., Fich, M., Mitchell, G. F., \& Moriarty-Schieven, G. 2001, ApJ, 559,307

Keene, J., Davidson, J. A., Harper, D. A., Hildebrand, R. H., Jaffe, D. T., Loewenstein, R. F., Low, F. J., \& Pernic, R. 1983, ApJ, 274, L43

Kelly, B. C. 2007, ApJ, 665, 1489

Kirk, J. M., Ward-Thompson, D., \& André, P. 2005, MNRAS, 360, 1506

Knacke, R. F., \& Thomson, R. K. 1973, PASP, 85, 341

Kramer, C., Alves, J., Lada, C., Lada, E., Sievers, A., Ungerechts, H., \& Walmsley, M. 1998, A\&A, 329, L33

Kramer, C., Richer, J., Mookerjea, B., Alves, J., \& Lada, C. 2003, A\&A, 399, 1073

Lamarre, J. M., et al. 1994, Infrared Phys. Technol., 35, 277

Martin, P. G., \& Whittet, D. C. B. 1990, ApJ, 357, 113

Mathis, J. S. 1990, ARA\&A, 28, 37

Mathis, J. S., Mezger, P. G., \& Panagia, N. 1983, A\&A, 128, 212

Mathis, J. S., Rumpl, W., \& Nordsieck, K. H. 1977, ApJ, 217, 425

Meny, C., Gromov, V., Boudet, N., Bernard, J.-P., Paradis, D., \& Nayral, C. 2007, A\&A, 468, 171

Motte, F., Andre, P., \& Neri, R. 1998, A\&A, 336, 150

Motte, F., Bontemps, S., Schilke, P., Schneider, N., Menten, K. M., \& Broguière, D. 2007, A\&A, 476, 1243

Mueller, K. E., Shirley, Y. L., Evans, N. J., II., \& Jacobson, H. R. 2002, ApJS, 143,469

Olofsson, S., \& Olofsson, G. 2009, A\&A, 498, 455

Ormel, C. W., Paszun, D., Dominik, C., \& Tielens, A. G. G. M. 2009, A\&A, 502,845
Ossenkopf, V. 1993, A\&A, 280, 617

Ossenkopf, V., \& Henning, T. 1994, A\&A, 291, 943

Pendleton, Y. J., Tielens, A. G. G. M., \& Werner, M. W. 1990, ApJ, 349, 107

Phillips, W. A. 1987, Rep. Prog. Phys., 50, 1657

Pollack, J. B., Hollenbach, D., Beckwith, S., Simonelli, D. P., Roush, T., \& Fong, W. 1994, ApJ, 421, 615

Press, W. H., Teukolsky, S. A., Vetterling, W. T., \& Flannery, B. P. 1992, Numerical Recipes in FORTRAN. The Art of Scientific Computing (Cambridge: Cambridge Univ. Press)

Rieke, G. H., \& Lebofsky, M. J. 1985, ApJ, 288, 618

Schlömann, E. 1964, Phys. Rev., 135, 413

Schnee, S., et al. 2010, ApJ, 708, 127

Shirley, Y. L., Evans, N. J., II, \& Rawlings, J. M. C. 2002, ApJ, 575, 337

Shirley, Y. L., Evans, N. J., II, Rawlings, J. M. C., \& Gregersen, E. M. 2000, ApJS, 131, 249

Shirley, Y. L., Mueller, K. E., Young, C. H., \& Evans, N. J., II 2003, in ASP Conf. Ser. 287, Galactic Star Formation Across the Stellar Mass Spectrum, ed. J. M. De Buizer \& N. S. van der Bliek (San Francisco, CA: ASP), 298

Shirley, Y. L., Nordhaus, M. K., Grcevich, J. M., Evans, N. J., II, Rawlings, J. M. C., \& Tatematsu, K. 2005, ApJ, 632, 982

Shu, F. H. 1977, ApJ, 214, 488

Stutz, A. M., et al. 2008, ApJ, 687, 389

Teixeira, P. S., Lada, C. J., \& Alves, J. F. 2005, ApJ, 629, 276

Tierney, L. 1998, Ann. Appl. Prob., 8, 1

Tomita, Y., Saito, T., \& Ohtani, H. 1979, PASJ, 31, 407

Tremaine, S., et al. 2002, ApJ, 574, 740

Visser, A. E., Richer, J. S., Chandler, C. J., \& Padman, R. 1998, MNRAS, 301, 585

Weiner, B. J., et al. 2006, ApJ, 653, 1049

Weingartner, J. C., \& Draine, B. T. 2001, ApJ, 548, 296

Whittet, D. C. B. 2003, Dust in the Galactic Environment (2nd ed.; Philadelphia, PA: Institute of Physics Publishing)

Whittet, D. C. B., Martin, P. G., Fitzpatrick, E. L., \& Massa, D. 1993, ApJ, 408, 573

Whitney, B. A., Wood, K., Bjorkman, J. E., \& Cohen, M. 2003, ApJ, 598, 1079

Wickramasinghe, N. C. 1967, The International Astrophysics Series (London: Chapman \& Hall)

Williams, S. J., Fuller, G. A., \& Sridharan, T. K. 2005, A\&A, 434, 257

Young, C. H., \& Evans, N. J., II. 2005, ApJ, 627, 293

Young, C. H., et al. 2004, ApJS, 154, 396

Zhou, S., Evans, N. J., II, Koempe, C., \& Walmsley, C. M. 1993, ApJ, 404, 232 